\title{
Высокие скорости требуют умных решений: кабельные системы и соединители Molex для передачи данных в дата-центрах и телекоммуникационной инфраструктуре
}

\author{
Р. Ромашко ${ }^{1}$, В. Ежов
}

УДК 621.315.21| ВАК 05.27.01

\begin{abstract}
Скорость передачи данных в современных дата-центрах
и телекоммуникационной инфраструктуре растет быстрыми темпами. Однако по мере увеличения скорости все более актуальной становится проблема ухудшения целостности сигнала, снижения отношения сигнал/шум. Из-за больших вносимых потерь на высоких частотах разработчики телекоммуникационного оборудования вынуждены переходить на менее экономически выгодные решения - оптоволокно или печатные платы из дорогостоящих материалов. Компания Molex - ведущий мировой производитель соединителей, кабельных сборок и других компонентов - предлагает передовые решения для передачи данных на скорости до 112 Гбит / с на основе меди в качестве проводника сигнала, которые позволяют снизить вносимые потери, сохранить целостность сигнала, эффективно управлять тепловыми режимами без изменения кабельной и сетевой инфраструктуры.
\end{abstract}

компании Molex значительный опыт создания соединительных систем для дата-центров, в состав которых входят серверы, сетевое оборудование (маршрутизаторы, серверы, роутеры), системы хранения данныхи другие компоненты. Среди новых решений Molex для высокоскоростной передачи данных - соединители и кабельные сборки на основе медных проводов, которые отличаются высокими характеристиками передачи сигналов при сохранении конкурентной цены. Рассмотрим ассортимент продуктов и решений, которые компания предлагает для этих приложений.

Наиболее перспективным на сегодняшний день методом модуляции сигналов для высокоскоростной передачи данных является модуляция РАМ-4, обеспечивающая скорость до 112 гбит / с. Реализация высокоскоростных каналов с использованием модуляции РАМ-4" требует снижения вносимых потерь и уровня помех в канале.

Компания «Золотой Шар», менеджер по продукции,

тел.: +7 495 234-01-10, romashko@zolshar.ru.

РАМ-4 - схема четырехуровневой импульсно-амплитудной моду-

ляции, передающая два бита на символ, что позволяет удвоить

скорость передачи данных по сравнению с традиционными

схемами кодирования "без возврата к нулю" (NRZ). Поскольку
Для решения этих задач компания Molex разработала кабельные сборки Bipass, которые позволяют снизить требования к целостности сигналов на основной печатной плате за счет передачи наиболее критичных высокоскоростных сигналов по твинаксиальному кабелю собственной торговой марки Теmp-Flex. Характеристики кабеля Temp-Flex и производство кабельных сборок на его основе в заводских условиях обеспечивают минимально допустимый фазовый сдвиг между сигналами дифференциальной пары.

Следует учитывать, что с внедрением высокоскоростной модуляции РАМ-4 увеличилась мощность, рассеиваемая специализированными ИС. В результате разработчики стали устанавливать на основных печатных платах или оптических трансиверах специальные устройства - ретаймеры, которые не только компенсируют уровень сигнала и восстанавливают тайминг в канале в соответствии с требованиями модуляции, но и позволяют уменьшить энергопотребление специализированных ИС. Однако такое решение лишь перераспределяет мощность, рассеиваемую в канале, с Ис на ретаймеры. Кабельные сборки

разница между уровнями сигнала РАМ-4 намного меньше, чем у NRZ, модуляция PAM-4 более восприимчива к шуму. 


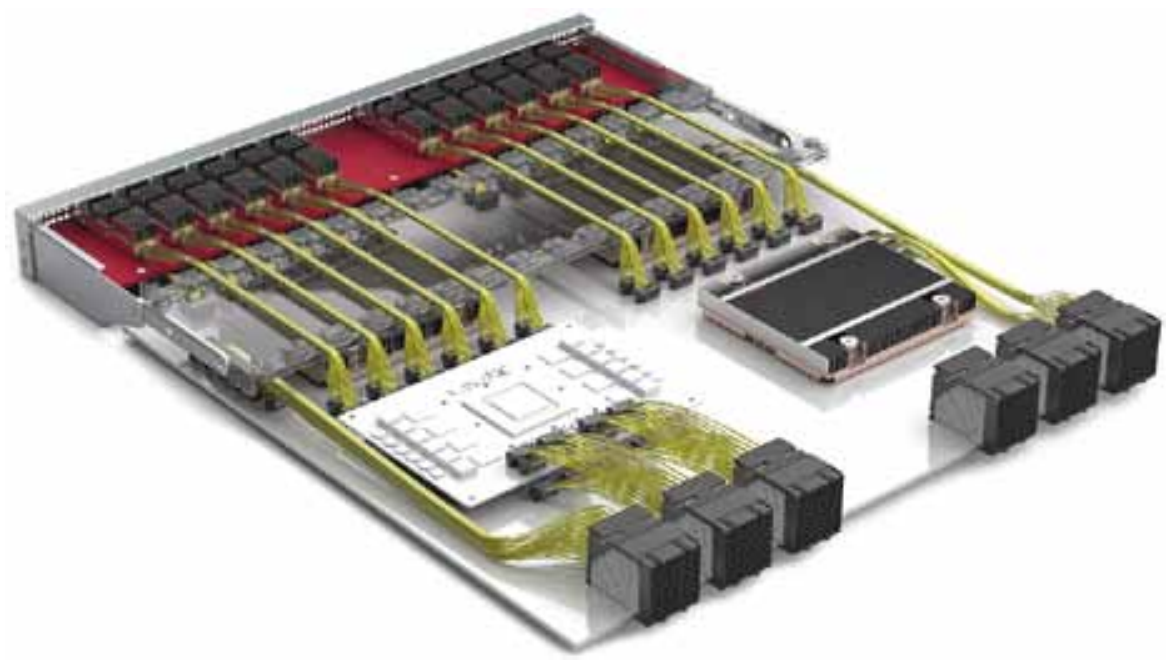

Рис. 1. Пример решения на основе системы BiPass с использованием разъемов QSFP-DD, Impulse и NearStack

BiPass, которые обеспечивают превосходную целостность сигнала, устраняют необходимость применения ретаймеров в канале, позволяют снизить энергопотребление специализированных ИС и улучшить условия теплообмена в оборудовании.

системы Bipass охватывают три категории соединений:

- между установленными на плате специализированными ИС и портами ввода-вывода;

- между разными специализированными ИС;

- между специализированными ИС и объединительной платой.

Пример решения на основе системы Bipass показан на рис. 1. В каждом из трех видов соединений системы BiPass значительно снижаются вносимые потери по сравнению с проводниками печатных плат.

Являясь более экономически выгодным решением, чем печатные платы, системы BiPass, кроме того, позволяют уменьшить число слоев и размеры печатных плат. Они обеспечивают гибкость проектного решения, поскольку ИС можно размещать на платах в любом месте.

Компания Molex предлагает также заказные шасси и панели на основе кабельных сборок BiPass, разработанные по требованиям клиента. Максимальная длина канала, построенная на базе пассивных кабельных сборок BiPass, может достигать 3 м.

кабельные сборки BiPass используют совместно с другими системами соединений Molex, в частности разъемами и кабельными системами стандарта QSFP-DD (Quad Small Form-Factor Pluggable Double Density), которые обеспечивают двойную плотность межсоединений по сравнению с широко применяемым сегодня стандартом QSFP (рис. 2). Системы QSFP-DD, совместимые c QSFP, обеспечивают передачу сигналов максимально для 256 дифференциальных пар по 32 портам со скоростью до 28 Гбит / с в формате NRZ или до 56 гбит/с в формате PAM-4. Таким образом, агрегатная скорость передачи данных по восьми каналам может достигать 200 или 400 Гбит/с. Причем формфактор разъемов QSFP-DD, шаг контактов в которых составляет 0,8 мм, остался таким же, как QSFP.

Система QSFP-DD совместима как с прежними кабельными системами, так и с разработанными в последнее время, поддерживает современные протоколы 200 Gigabit Ethernet u InfiniBand 100 Gigabit (EDR), a тakже 10 Glops Ethernet, 14 Gbps (FDR) InfiniBand и 16 Glops Fibre Channel.

QSFP-DD предназначены для серверов, сетевых ключей, маршрутизаторов и SAN / NIC-карт. Опционально доступны варианты на 2/4/8 каналов, каждый из которых работает в полнодуплексном режиме передачи данных. Кабельные системы QSFP-DD coвместимы со спецификациями MSA, IEEE, SFF, способны работать в тяжелых условиях дата-центров при температуре от -20 до $85^{\circ} \mathrm{C}$. Разъемы QSFP-DD доступны в исполнении для поверхностного монтажа, предлагается также опция для размещения разъемов с двух сторон печатной платы.

Для защиты от электромагнитных помех разъемы QSFP-DD помещают в корпус из нержавеющей стали, который обеспечивает повышенную надежность и прочность. Для более эффективного отвода тепла от ИС или модулей на корпусах разъемов QSFP-DD монтируются радиаторы с никелированным покрытием (рис. 3).

Полностью интегрированное решение QSFP-DD от Molex включает в себя корпус разъема, кабель и смонтированные печатные платы. Доступны интегрированные

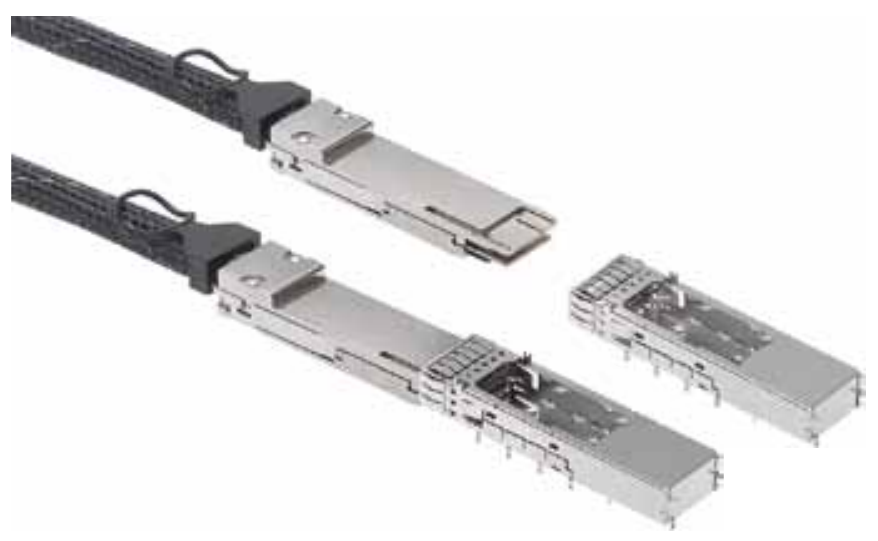

Рис. 2. Кабельная сборка стандарта QSFP-DD 


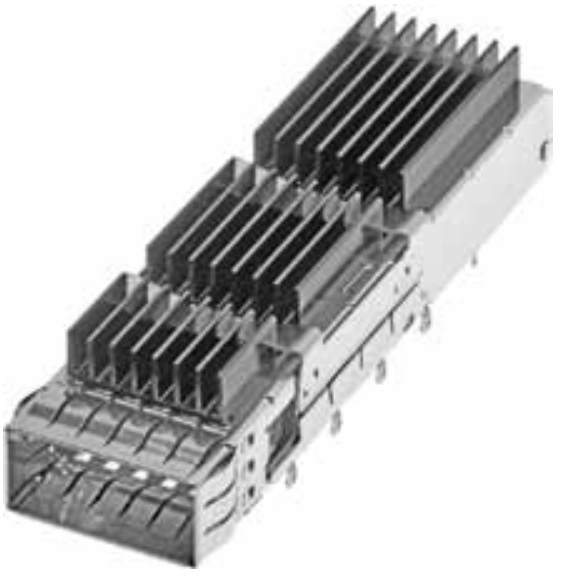

Рис. 3. Корпус

разъема

QSFP-DD из

нержавеющей

стали

с радиаторами

разъемы и защитные корпуса стандарта QSFP-DD в многоярусном исполнении в конфигурации $1 \times 1$ или $2 \times 1$.

Для современных дата-центров, где наряду с высокой скоростью передачи данных требуется высокая плотность монтажа, компания Molex разработала семейство разъемов Impulse для объединительных плат (рис. 4), которое поддерживает скорость передачи 56 гбит/с (NRZ) или 112 гбит / с (PAM-4) и обеспечивает высокую целостность сигнала. Благодаря прямой ортогональной конструкции разъемов отпадает необходимость в промежуточных объединительных платах, что снижает общую стоимость системы и улучшает условия отвода тепла за счет воздушного охлаждения. Компактные размеры и высокая плотность размещения контактов достигаются благодаря малому шагу сетки контактов - всего $2 \times 4$ мм

Инновационная конструкция разъемов обеспечивает снижение вносимых потерь по сравнению с традиционными линейными конструкциями, смещая частоту резонанса интерфейса далеко за пределы 30 ГГц. Эти разъемы позволяют масштабировать коммуникационное оборудование, что минимизирует затраты при расширении инфраструктуры дата-центров. Семейство Impulse отвечает перспективным требованиям к скорости передачи данных, что позволит в будущем обойтись без изменения конфигурации оборудования. В кабельных сборках Impulse применяются высококачественные кабели торговой маркитеmp-Flex от Molex.
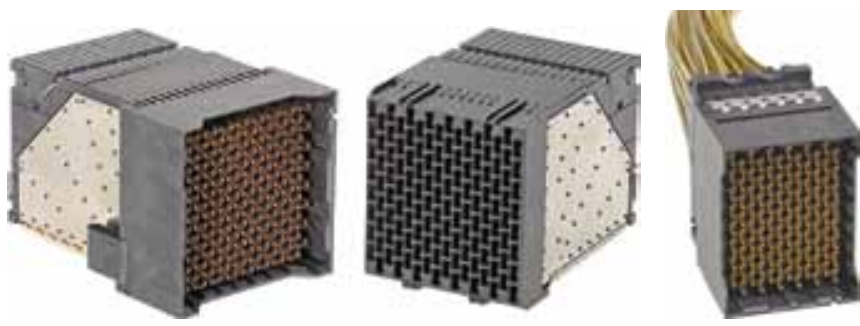

Рис. 4. Разъемы Impulse
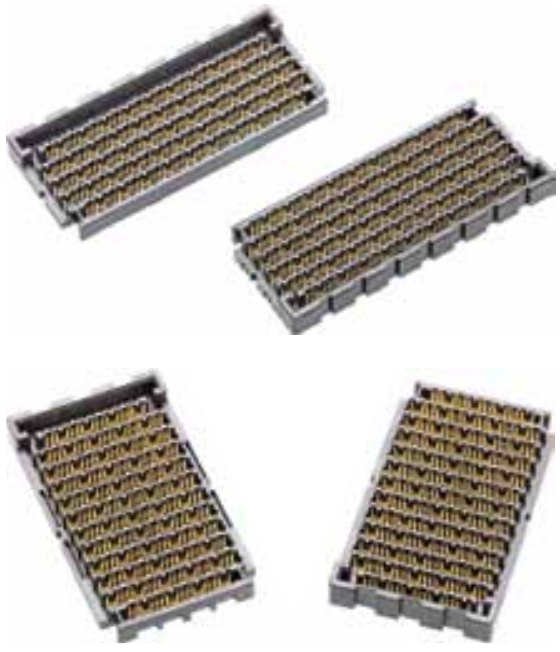

Рис. 5.

Варианты конфигурации разъемов Mirror Mezz

Все сигнальные контакты разъемов Impulse установлены в отдельных экранированных гнездах. Прочные направляющие обеспечивают точное выравнивание и сопряжение разъема, а U-образные ножевые заземляющие контакты позволяют механически изолировать сигнальные контакты, снизить риск искривления контактов при эксплуатации разъемов.

Еще одно семейство высокоскоростных соединителей Molex - гермафродитные разъемы Mirror Mezz, которые поддерживают скорость передачи до 56 Гбит / с на дифференциальную пару, обеспечивают снижение затрат в серверных приложениях, поскольку отпадает необходимость в дополнительных ответных частях соединительных систем (потребуется только два одинаковых разъема, которые соединяются между собой). Максимальная гибкость проектного решения достигается благодаря тому, что пользователь может соединять гермафродитные разъемы различного вертикального размера (2,5 или 5,5 мм) и получать желаемую для своего приложения высоту сопряжения (5, 8 или 11 мм).

Разъемы Mirror Mezz обеспечивают очень высокую плотность контактов, например, с помощью разъема $15 \times 11$ ОСР можно достичь плотности в 165 дифференциальных пар на квадратный дюйм (рис. 5). В разъемах применена особая структура контактного узла, которая обеспечивает надежный 2-точечный контакт, предотвращает вибрацию и сдвиг контактов. Форма золоченых контактов гарантирует надежное соединение в жестких условиях эксплуатации, номинальная величина проскальзывания контактдеталей составляет 1,5 мм для достаточного сцепления.

Прочная конструкция корпуса разъема, гарантирующая защиту контактов, содержит направляющие элементы, исключающие любую возможность неправильного соединения. Широкие земляные контакты обеспечивают равномерное распределение электрического поля и защищают сигналы дифференциальных пар от влияния соседних линий передачи. 

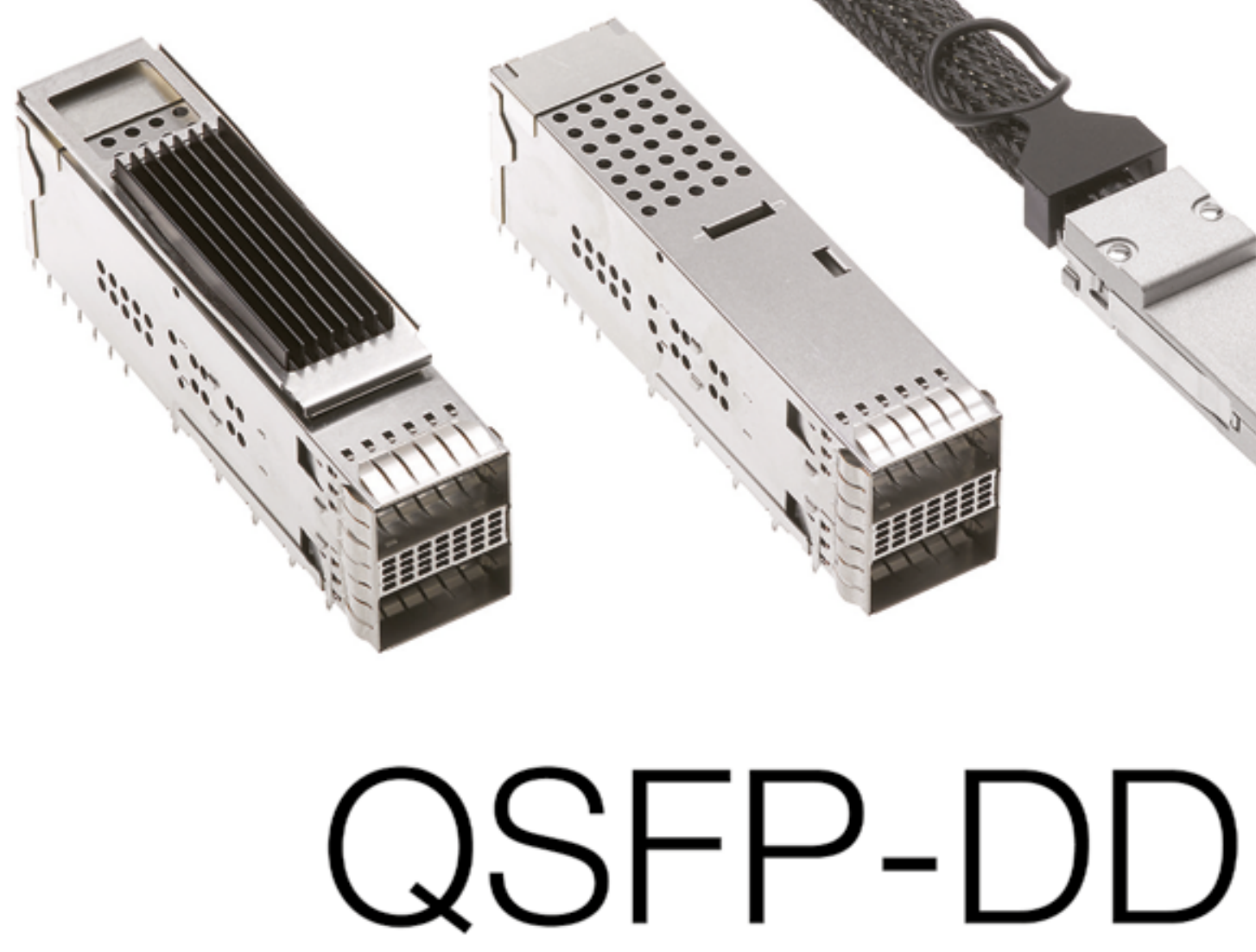

Разъёмы и кабельные сборки двойной плотности

\section{Плотность ×2}

\section{Скорость до 400 Гбит/с}

\section{Вносимые потери <0,8 дБ}

$$
\text { от }-20^{\circ} \text { до }+85^{\circ} \mathrm{C}
$$

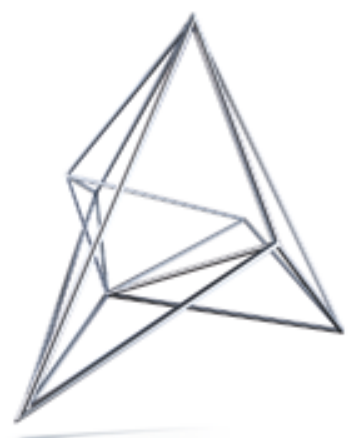

\section{Symmetron}

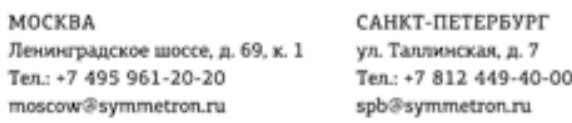

Ter: + 7 495 961-20-20 Ter: +7 812 449-40-0

www.symmetron.ru

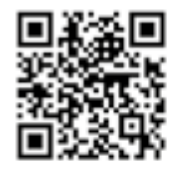

www.symmetron_ru/400gb

тех. поддержка:

molex@symmetron.ru

НОВОСИБИРСК

ул. Блхохера, д. 716

Ter: +7383 361-34-24

sibirisymmetron.ru
Минск

ул. В. Хоружей, д. 1a, 0 ф. 403

Ter: +375 17 336-06-06

minsk:symmetron.rs 


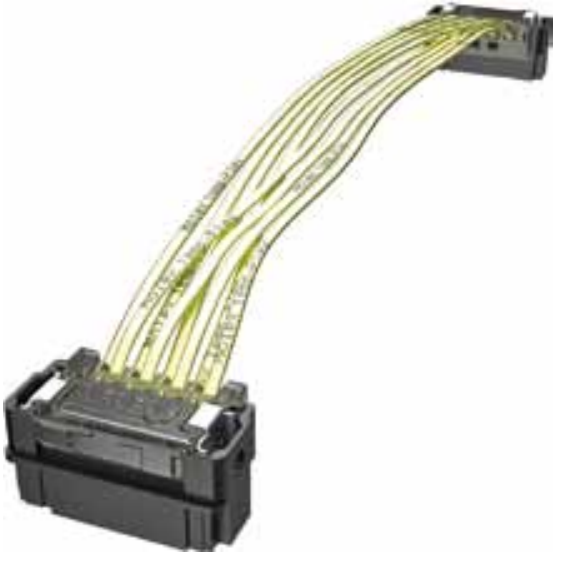

Рис. 6.

Кабельная

сборка

NearStack

Разъемы Mirror Mezz монтируются на плату с помощью BGA-шариков, причем используется прошитая (stitched) структура размещения ВСА-шариков, чтобы повысить технологичность и сократить время изготовления изделий. В семействе представлены версии с различными числом и конфигурацией контактов, например, 6 дифференциальных пар х 5 рядов, 6 дифференциальных пар × 15 рядов, 10 дифференциальных пар × 11 рядов и др.

Среди соединительных систем Molex для высокоскоростной передачи данных стоит отметить недавно разработанное семейство разъемов и кабельных сборок Nearstack на основе твинаксиального кабеля Temp-Flex, которое обеспечивает скорость до 56 Гбит / с (NRZ), а в перспективе - до 112 Гбит / с при использовании модуляции РАМ-4 (рис. 6). Они применяются для прямого соединения точек на плате или между платами, чтобы исключить нарушение непрерывности полного сопротивления, высокие вносимые потери, влияние перекрестных помех, а также уменьшить задержку сигналов. Такое решение, позволяющее отказаться от ретаймеров и использовать печатные платы с меньшим числом слоев или меньшей площади, снижает общую стоимость системы.

В разъемах NearStack шаг контактов составляет всего 0,6 мм, их высота - 9 мм, что обеспечивает высокую плотность размещения сигнальных контактов (до 30 дифференциальных пар на квадратный дюйм) и позволяет использовать их в условиях ограниченного объема. Допустимо размещать на печатной плате несколько рядов разъемов NearStack около специализированной ИС. Специальная луженая шпилька разъемов обеспечивает надежное соединение с платой.

Минимальная длина кабелей в системе NearStack coставляет 150 мм, а максимальная -1,5 м. Доступна заказная длина кабеля по требованиям клиента. Специальные вырезы на разъемах NearStack гарантируют правильное сопряжение разъема с ответной частью. Для них допустим автоматизированный процесс установки на печатную плату.

Кабельные сборки NearStack доступны и с другими типами разъемов Molex на противоположном

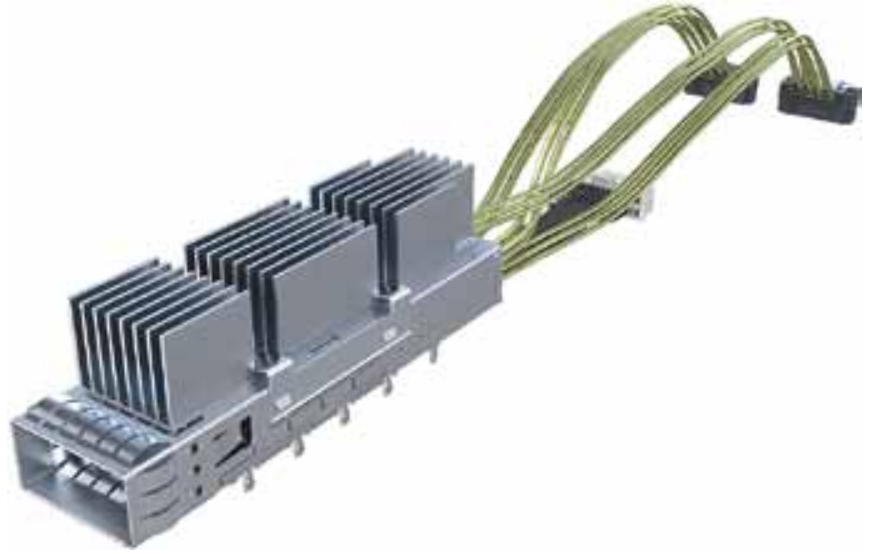

Рис. 7. Вариант комбинированной кабельной сборки с разъемами NearStack и QSFP-DD

конце кабеля, например с разъемами QSFP-DD или Impulse, чтобы обеспечить оптимальное решение для соединения точек на плате с портами ввода-вывода или с объединительной платой (рис. 7). Опционально для разъемов Nearstack доступен зажим с петлей для размыкания разъемов. Зажим блокирует защелки для предотвращения самопроизвольного размыкания разъема.

Минимальный ресурс разъемов Nearstack составляет 100 циклов, сопротивление контактов - 20 мОм, диапазон рабочих температур - от -55 до $105^{\circ} \mathrm{C}$.

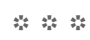

Bсе решения компании Molex для высокоскоростной передачи данных являются масштабируемыми и обеспечивают максимальную гибкость проекта. Они не требуют изменения конфигурации существующей сети и в то же время отвечают перспективным требованиям по скорости, сохраняют высокую целостность сигнала и отличаются оптимальными характеристиками канала передачи данных. Продукция Molex проходит 100\%-ное тестирование, поэтому пользователю необязательно проводить входной контроль изделий.

По вопросам приобретения продукции Molex обращайтесь в холдинг "Золотой Шар» (www.zolshar.ru).

\section{ЛИТЕРАТУРА}

1. Support Tomorrow's Speeds Inside Today's Footprint. Molex Solutions for 112 Gbps Architecture // www.molex.com

2. Molex Bipass I/ O And Backplane High-Speed Solutions // www.molex.com

3. QSFP-DD (Double Density) Interconnect System and Cable Assemblies // www.molex.com

4. NearStack High-Speed Connector System and Cable Jumper Assemblies // www.molex.com

5. Direct Attach Cables For Data Centers // www. molex.com 